\title{
0 idoso e sua espiritualidade: impacto sobre diferentes aspectos do envelhecimento
}

The elderly and their spirituality: impact on different aspects of aging

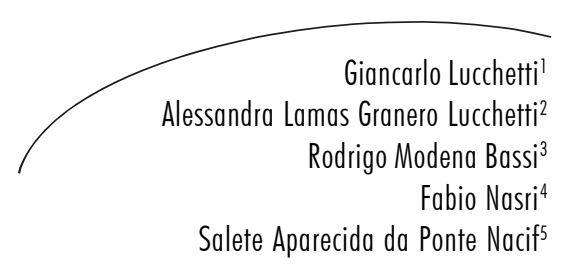

Resumo

Introdução: $\mathrm{O}$ interesse sobre a espiritualidade e a religiosidade sempre existiu no curso da história humana, a despeito de diferentes épocas ou culturas. Contudo, apenas recentemente a ciência tem demonstrado interesse em investigar o tema. Estudos e pesquisas demonstram que a faixa etária acima dos 65 anos é aquela com mais contato frente a sua espiritualidade e religiosidade. $\mathrm{O}$ objetivo do presente estudo é realizar uma revisão da literatura científica que norteia essa temática e investigar qual o impacto da espiritualidade em diferentes aspectos do envelhecimento. Metodologia: Pesquisa nas bases de dados Pubmed/Medline, LILACS e Scielo, nos idiomas inglês e português, entre 1966 e 2009, utilizando as palavras-chave aged/idoso e spirituality/espiritualidade. Resultados: Foram incluídos 48 artigos divididos nas seguintes temáticas: envelhecimento bem-sucedido, bemestar, qualidade de vida, doenças crônico-degenerativas, doenças neuropsiquiátricas, funcionalidade, mortalidade e impacto no fim da vida. Conclusão: Conclui-se que o envelhecimento possui uma relação íntima com a espiritualidade nos seus mais diferentes aspectos e percebe-se que há uma escassez de pesquisas sobre espiritualidade/religiosidade em idosos.

\section{Abstract}

The interest on spirituality and religion has always existed in the course of human history, despite different periods or cultures. However, only recently, science has shown interest in investigating this issue. Studies demonstrate that

\footnotetext{
Setor de Geriatria da Irmandade da Santa Casa de Misericórdia de São Paulo e Grupo de Estudos em Envelhecimento da Associação Médico-Espírita de São Paulo. São Paulo, SP, Brasil.

2 Centro Interdisciplinar de Assistência e Pesquisa em Envelhecimento, Faculdade de Ciências Médicas de Minas Gerais e Grupo de Estudos em Envelhecimento da Associação Médico-Espírita de São Paulo. São Paulo, SP, Brasil.

3 Grupo de Estudos em Envelhecimento da Associação Médico-Espírita de São Paulo. São Paulo, SP, Brasil.

4 Programa de Geriatria e Gerontologia do Hospital Albert Einstein e Grupo de Estudos em Envelhecimento da Associação Médico-Espírita de São Paulo. São Paulo, SP, Brasil.

5 Disciplina de Clínica Médica da Universidade Federal de São Paulo e Grupo de Estudos em Envelhecimento da Associação Médico-Espírita de São Paulo. São Paulo, SP, Brasil.
}

Palavras-chave:

Espiritualidade. Geriatria. Idoso. Religião. 
those aged 65 years old or more have more contact with their spirituality and religiosity. This article aims to conduct a review from scientific literature that deals with this issue and investigate the impact of spirituality on different aspects of ageing. Methods: A search on Pubmed/Medline, LILACS and Scielo was carried out, including articles from 1966 to 2009, in English or Portuguese, using the keywords: aged/idoso e spirituality/espiritualidade. Results: Forty eight articles were included and divided in: successful aging, well-being, quality of life, chronicdegenerative diseases, neuro-psychiatric diseases, functional status, mortality and end of life care. Conclusion: Aging has a close relationship with spirituality in many different aspects and there is a paucity of research on spirituality/religiosity in the elderly.

\section{INTRODUÇÃO}

O envelhecimento populacional é uma realidade mundial. Em países desenvolvidos, a população idosa (acima de 65 anos) já ultrapassou o número de crianças e estima-se que em 2050 teremos $32 \%$ de pessoas idosas, totalizando uma criança para cada dois idosos. ${ }^{1}$

De acordo com Cavalcante et al., " "o fato de a velhice ser considerada a última etapa da vida faz com que ocorra um aumento da frequência sobre o pensar na morte e, sobretudo, a respeito do que vem depois dela. Se a questão da finitude parecia longínqua, pouco pensada, na velhice, torna-se mais próxima e até real. A morte de pais, parentes e amigos remete imediatamente à própria morte. O retorno a uma prática religiosa passa a ser mais evidente, sendo por muitos percebida como indispensável. Não é sem razão que muitos consideram a velhice como a etapa em que um balanço da vida é necessário e inevitável”.

\section{DEFINIÇÕES}

Inicialmente, faz-se necessária uma discussão pormenorizada sobre os conceitos básicos de religião, religiosidade e espiritualidade. Segundo Koenig et al., no livro Handbook of Religion and Health. $^{3}$

- Religião é o sistema organizado de crenças, práticas, rituais e símbolos designados para facilitar o acesso ao sagrado, ao
Key words: Spirituality. Religiousness. Geriatrics. Elderly. transcendente (Deus, força maior, verdade suprema ...).

- Religiosidade é o quanto um indivíduo acredita, segue e pratica uma religião. Pode ser organizacional (participação na igreja ou templo religioso) ou não-organizacional (rezar, ler livros, assistir programas religiosos na televisão).

- Espiritualidade é uma busca pessoal para entender questões relacionadas à vida, ao seu sentido, sobre as relações com o sagrado ou transcendente que podem ou não levar ao desenvolvimento de práticas religiosas ou formações de comunidades religiosas.

\section{EPIDEMIOLOGIA DA RELIGIÃO NO IDOSO}

De acordo com a pesquisa promovida pelo Instituto Gallup Internacional em $2005,{ }^{4}$ que envolveu cerca de 50.000 pessoas em 65 países do mundo, mais de um terço dos entrevistados revelaram ser religiosos e os idosos foram aqueles com maior grau de religiosidade (quase $70 \%$ declararam-se religiosos, em oposição a $60 \%$ dos jovens). Resultado semelhante já havia sido identificado por McFadden em $1995,{ }^{5}$ em que os idosos mostraram maior envolvimento religioso.

No Brasil, de acordo com o Censo Demográfico de 2000, ${ }^{6}$ os idosos sem nenhum tipo de religião perfaziam 3,6\% do total, contrapondo valores de até $8,0 \%$ para faixas etárias mais jovens. 
Apesar disso, ainda há poucas pesquisas mundiais envolvendo saúde e espiritualidade/ religiosidade em populações exclusivamente idosas. No presente estudo, objetiva-se realizar uma revisão da literatura científica que norteia essa temática e investigar qual o impacto da espiritualidade em diferentes aspectos do envelhecimento.

\section{METODOLOGIA}

O estudo constituiu-se de uma revisão bibliográfica através de busca nos bancos de dados Pubmed/Medline, LILACS e SciELO, nas línguas portuguesa e inglesa. Utilizaram-se, para a busca, as seguintes palavras-chave: aged/idoso e spirituality/ espiritualidade. Na busca, no período de 1966 a 2009; 1.336 artigos foram publicados no Pubmed, 1.253 no LILACS e três artigos na SciELO. Nesta fase, foi realizada leitura exploratória dos títulos e resumos dos estudos, com o reconhecimento do material que atenderia aos critérios de inclusão do estudo.

Para inclusão, os artigos deveriam possuir qualidade técnica, definida pelos autores desta revisão, e possuir relação com o impacto na vida do idoso, incluindo as seguintes subdivisões: envelhecimento bem-sucedido, bem-estar e qualidade de vida, doenças crônico-degenerativas, doenças neuro-psiquiátricas, funcionalidade, mortalidade, cuidados no fim da vida/finitude e aspectos negativos da religião na saúde do idoso.

\section{RESULTADOS E DISCUSSÃO}

$\mathrm{Na}$ busca bibliográfica final, foram obtidos 105 resultados, que foram lidos na íntegra para uma amostra final de 48 artigos, ${ }^{7-8,11-17,19-57}$ que possuíam maior relevância para cada subdivisão.
Foram incluídas também uma tese de doutorado 9 e uma citação de $\operatorname{livro}^{10}$ para a subdivisão "envelhecimento bem-sucedido".

\section{Impacto no Envelhecimento bem-sucedido}

Envelhecimento bem-sucedido conforme proposto por Rowe \& Kahn ${ }^{7}$ em 1997, inclui três elementos: probabilidade baixa de doenças e de incapacidades relacionadas às mesmas; alta capacidade funcional cognitiva e física; e engajamento ativo com a vida.

Alguns estudos nacionais têm demonstrado que o impacto das crenças pessoais influencia no envelhecimento bem-sucedido. Em 2005, estudo conduzido no Rio Grande do Sul por Moraes e $\mathrm{cols}^{8}$ mostrou que aqueles idosos cujas crenças pessoais davam maior significado a suas vidas tinham até dez vezes mais chance de cursar com envelhecimento bem-sucedido, em comparação com aqueles que não as possuíam. Outro estudo gaúcho realizado por Rosa em $2008^{9}$ mostrou que a resiliência (capacidade humana muito presente em pacientes com bem-estar espiritual, que consiste em enfrentar, vencer e ser fortalecido ou transformado nas experiências de adversidade) foi associada ao envelhecimento bem-sucedido.

Da mesma forma, estudo internacional realizado por Wong ${ }^{10} \mathrm{em} 1998$ demonstrou que as atividades religiosas, o coping religioso (modo de lidar com a doença utilizando-se da religião) e o maior significado à vida eram preditores daqueles que envelheceram de maneira bem-sucedida ou não. Seguindo essa tendência, Crowther et al. ${ }^{11}$ propuseram uma alteração no modelo de envelhecimento saudável de Rowe \& Kahn, ${ }^{7}$ que seria a introdução de um quarto fator: a espiritualidade, conforme visualizado na figura 1. 


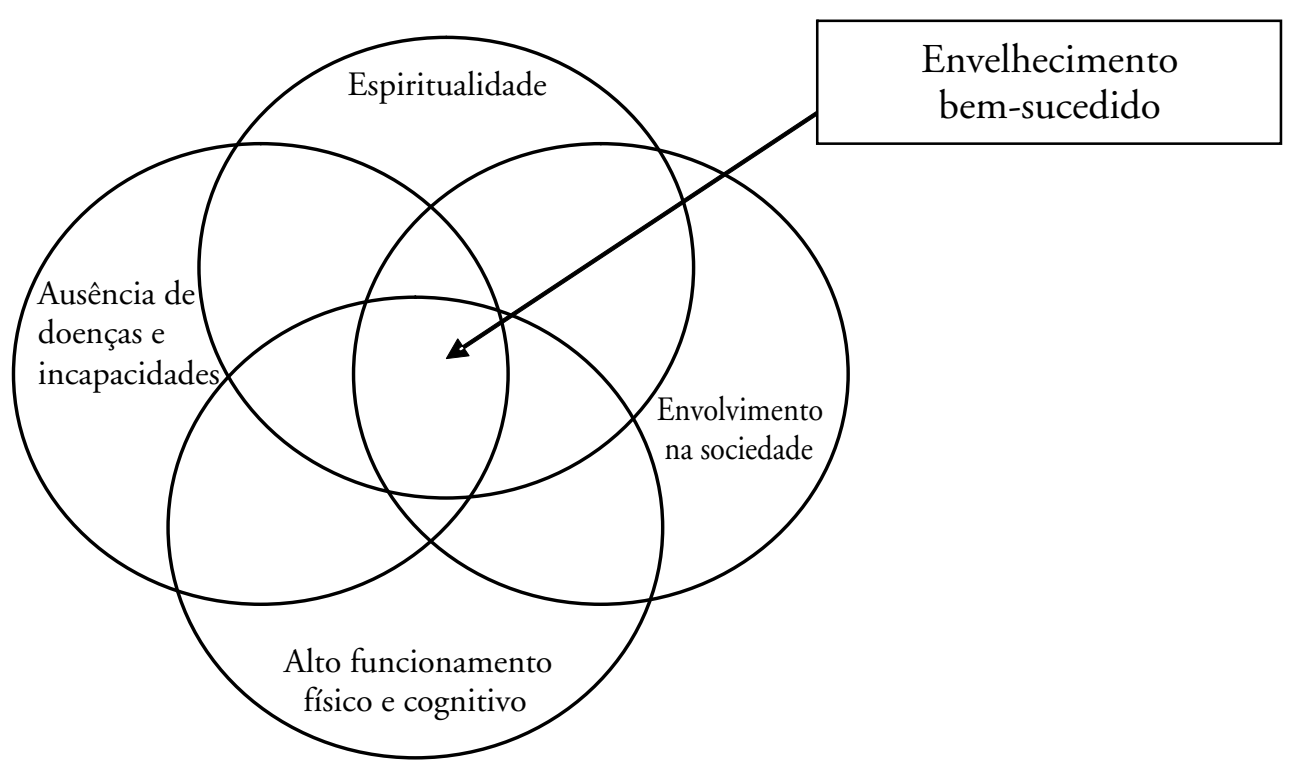

Figura 1 - Modelo de envelhecimento bem-sucedido proposto por Rowe and Kahn e modificado por Crowther et al, 2002. ${ }^{11}$

Impacto no bem-estar e qualidade de vida

O impacto na qualidade de vida tem sido demonstrado de forma quantitativa e qualitativa. Claro exemplo disso foi um inquérito populacional conduzido no município de Botucatu-SP envolvendo 365 idosos. ${ }^{12}$ Estes foram questionados sobre o que era qualidade de vida e a resposta "ter religião e fé" foi a sétima mais comum.

Em 2005, ${ }^{13}$ foi realizada uma meta-análise dos principais estudos que envolviam o tema espiritualidade e qualidade de vida. $\mathrm{Na}$ análise final, houve uma correlação moderada $(\mathrm{r}=0.34$, 95\% CI: 0.28-0.40) entre níveis mais altos de espiritualidade/religiosidade e melhor qualidade de vida.

Em 2003, Katsuno ${ }^{14}$ demonstrou que a espiritualidade estava correlacionada à melhor qualidade de vida subjetiva em pacientes com demência leve. Da mesma forma, outros estudos têm demonstrado uma associação direta entre maior frequência religiosa e maior satisfação com a vida, ${ }^{15}$ incluindo um estudo envolvendo 709 pessoas acima de 55 anos que participaram do
World Values Survey. ${ }^{16}$ Com a mesma temática, estudo realizado na China demonstrou íntima relação entre qualidade de vida e espiritualidade em idosos com deficiências visuais. ${ }^{17}$

\section{Impacto nas doenças crônico-degenerativas}

Segundo a Organização Pan-Americana de Saúde (OPAS),${ }^{18}$ as doenças crônico-degenerativas são definidas como doenças não-transmissíveis, que incluem, dentre outras, osteoartrite, osteoporose, hipertensão, coronariopatia, diabetes, hipotireoidismo.

Quanto às doenças osteoarticulares, os idosos com artrite que cultivavam um maior número de experiências espirituais diárias possuíam maior energia e menor depressão. ${ }^{19} \mathrm{Da}$ mesma forma, em pacientes com artrite reumatóide, aqueles com maior espiritualidade tiveram maior resiliência e percepção de saúde..$^{20,21}$

Nas doenças cardiovasculares, Hummer et al. ${ }^{22}$ avaliaram uma amostra representativa da população americana de 21.204 pessoas durante oito anos, e mesmo após controlar para aspectos sociais, físicos e sociodemográficos, a maior 
frequência religiosa esteve associada a uma menor mortalidade por causas cardiovasculares. Em 2007, Bekelman et al. ${ }^{23}$ demonstraram correlação entre maior espiritualidade e menor depressão em pacientes idosos com insuficiência cardíaca, resultado este que foi semelhante ao obtido em uma população iminentemente geriátrica internada por acidente vascular cerebral. ${ }^{24}$

Quanto à hipertensão, apesar da dificuldade de encontrar estudos somente envolvendo a população geriátrica, uma subdivisão do banco de dados NHANES III demonstrou que aqueles que frequentavam pelo menos uma vez por semana a igreja ou templo religioso tinham menor pressão arterial sistólica estatisticamente significante, quando comparados com os que não frequentavam, inclusive quando ajustados para a idade, ${ }^{25}$ dado este de acordo com outro estudo americano. ${ }^{26}$

Em um estudo japonês envolvendo somente idosos, os idosos mais espiritualizados reportaram menos hipertensão que os demais. ${ }^{27}$

\section{Impacto nas doenças neuro-psiquiátricas}

No campo das doenças neuro-psiquiátricas, a depressão é talvez a doença que tenha maior correlação com as crenças religiosas e espirituais. Estudos demonstram maior prevalência de depressão em idosos não-religiosos ou nãoespiritualizados portadores de neoplasias, ${ }^{28} \mathrm{em}$ reabilitação, ${ }^{29} \mathrm{em}$ pacientes da comunidade ${ }^{30,31} \mathrm{e}$ em pacientes hospitalizados. ${ }^{32} \mathrm{Da}$ mesma forma, há uma maior remissão da depressão quanto maior a espiritualidade do idoso. ${ }^{33,34}$

Com relação à ansiedade, estudos demonstram que quanto maior a religiosidade extrínseca (frequência religiosa), maior o grau de ansiedade, porém quanto maior a religiosidade intrínseca (crenças interiorizadas como parte integrante de sua vida), menor o grau de ansiedade. ${ }^{35,36}$ Segundo Koenig ${ }^{3}$, a ansiedade trazida pelo medo da morte parece diminuir à medida que o idoso é mais espiritualizado.

Já na doença de Alzheimer, alguns estudos têm demonstrado a influência da espiritualidade nas síndromes demenciais. Estudo recente publicado na revista Neurology $y^{37}$ demonstrou que altos níveis de espiritualidade e práticas religiosas foram associados a uma menor progressão da doença de Alzheimer. Este achado, que já havia sido investigado por Hill et al. ${ }^{38}$ em 2006, mostrou que a frequência religiosa foi associada a menores taxas de declínio cognitivo, ao avaliar cerca de 3.000 pacientes idosos. Além disso, a prece e a leitura da Bíblia já têm se mostrado alternativas para pacientes demenciados agitados, ${ }^{39,40}$ assim como atividades religiosas e espirituais têm sido relatadas como capazes de prevenir agitação. ${ }^{41}$

Os estudos com doença de Parkinson são escassos na literatura. $\mathrm{Na}$ busca realizada, foi encontrado um estudo em que os autores fizeram entrevistas com os pacientes com doença de Parkinson e seus familiares. $\mathrm{Na}$ análise final, as crenças religiosas e a fé influenciaram na forma de lidar com a doença. ${ }^{42}$

\section{Impacto na funcionalidade}

Alguns estudos apontam para relações entre religiosidade / espiritualidade e funcionalidade em idosos. Estudo realizado em 2004 mostrou que o coping religioso foi preditivo da evolução física e funcionalidade dos pacientes idosos hospitalizados. ${ }^{43}$ No mesmo ano, Koenig et al. ${ }^{44}$ mostraram uma relação fraca entre religiosidade e espiritualidade com a saúde física do idoso.

Da mesma forma, outro estudo avaliou idosas admitidas para correção de fratura de quadril e demonstrou uma correlação entre crenças religiosas, menor depressão e melhor recuperação para deambulação no momento da alta hospitalar. ${ }^{29}$

Finalmente, Kim et al., ${ }^{45} \mathrm{em}$ 1998, mostraram uma correlação positiva entre espiritualidade e funcionalidade em adultos em reabilitação.

Impacto na mortalidade

O impacto na mortalidade vem sendo avaliado de forma consistente nos últimos anos. Em estudo 
realizado por Kenneth em 2001, ${ }^{46}$ houve relação entre sofrimento religioso (pensamentos de que Deus o abandonou, questionamentos do amor de Deus) e aumento de mortalidade, com um risco relativo (RR) de 1,19 (1,05-1,33).

Algumas meta-análises têm sido elaboradas, mostrando uma relação entre maior espiritualidade e religiosidade com maior sobrevida. ${ }^{47,48}$ Em 2001, McCullough et. al. ${ }^{48}$ demonstraram uma menor taxa de mortalidade para aqueles que frequentavam serviços religiosos uma vez por semana ou mais, em comparação com aqueles que frequentavam menos. De acordo com os autores, "elucidar a natureza desta associação robusta porém pouco compreendida pode ser um campo fértil para os pesquisadores".

E por último, estudo realizado por Hall, em $2006,{ }^{49}$ fez uma comparação audaciosa entre a custo-efetividade do uso de estatinas e da frequência religiosa. O título da publicação foi "Frequência religiosa: mais efetiva que Lipitor ${ }^{\mathrm{TM}}$ " e fez uma comparação sugerindo que a frequência religiosa seria mais custo-efetiva que as estatinas.

\section{Impacto no fim da vida}

As necessidades espirituais crescem de significado à medida que se aproxima a finitude. Estudo realizado em pacientes terminais demonstrou que os assuntos espirituais são muito importantes nessa fase. ${ }^{50}$ Outro estudo mostrou que $94 \%$ dos pacientes gostariam de ser questionados sobre questões espirituais se estivessem gravemente doentes. ${ }^{51}$

Em 2003, McClain et al. ${ }^{52}$ mostraram que o bem-estar espiritual protegeu do desespero do fim da vida em pacientes em cuidados paliativos. Segundo Puchalsky et al., ${ }^{53}$ pacientes terminais devem ter a oportunidade de encontrar seu significado de vida, amor e companheirismo, o que deveria ser feito por meio de uma abordagem espiritual.
A importância deste tema viria pelo fato de que os pacientes utilizam-se de suas crenças religiosas e espirituais para decisões sobre medidas suportivas de vida, e alguns pacientes podem ter um sofrimento espiritual muito importante e a própria espiritualidade pode auxiliar o paciente a um menor sofrimento, menor valorização da dor e maior aceitação da morte..$^{54-56}$

\section{Aspectos negativos da religião na saúde}

Do mesmo modo que a religiosidade pode estar positivamente associada à saúde do paciente, alguns estudos apontam seus aspectos negativos. Koenig et al., em 2001, ${ }^{46}$ realizaram um estudo coorte em que aqueles que possuíam dúvidas e conflitos religiosos apresentaram maior mortalidade. Esse achado é semelhante a outro trabalho realizado em pacientes com mieloma múltiplo, em que o conflito religioso foi preditor de maiores taxas de depressão, estresse, fadiga e dor. ${ }^{57}$

Os sentimentos mais comuns envolvidos com esses aspectos negativos são: descontentamento espiritual, pensamento de que Deus está punindo e questionamento dos poderes de Deus. ${ }^{58}$

\section{CONCLUSÃO}

Conclui-se que o envelhecimento possui uma relação íntima com a espiritualidade nos mais diferentes aspectos do envelhecimento, tendo impacto desde o envelhecimento bem-sucedido até os cuidados no fim da vida.

Apesar de ser um dos grupos em que a espiritualidade tenha maior relevância, percebese que ainda há uma escassez de pesquisas sobre espiritualidade / religiosidade em idosos.

O profissional de saúde que lida com o paciente geriátrico deve estar treinado a abordar esta questão e atento a seus aspectos positivos e negativos, de forma a ajudar o idoso nesta fase da vida, respeitando suas escolhas e individualidade. 


\section{REFERÊNCIAS}

1. World Health Organization. World Population Prospects: The 2004 Revision. [acesso em: 31mar 2009]; Disponível em: http://www.un.org/esa/ population/publications/WPP2004/ 2004Highlights_finalrevised.pdf.

2. Cavalcante AM. Psiquiatria on line Brasil: a psicologia do idoso.[ Acesso em $11 \mathrm{fev}$ 2009] .Disponível em: http://www.polbr.med.br/ arquivo/mour0502.htm

3. Koenig HG, Mccullough ME, Larson DB. Handbook of Religion and Health. New York: Oxford University Press; 2001. 712.

4. James M. Voice of the people 2005 : religiosity around the world. Gallup International.[ Acessado em: 10 de fev de 2009] Disponível em: http://extranet.gallup-international.com/ uploads/internet/

Religiosity\%20around\%20the\%20world\% 20VoP\%2005\%20press\%20release.pdf.

5. McFadden SH. Religion and well being in aging persons in an aging society. Journal of Social Issues 1995; 51 (2): 161-175.

6. Instituto Brasileiro de Geografia e EstatísticaCenso Demográfico - 2000 : Características Gerais da População. Censo Demográfico 2000: Características gerais da população. [Acesso em $10 \mathrm{Fev}$ 2009]. Censo Demográfico - 2000 : Características Gerais da PopulaçãoCenso Demográfico - 2000 : Características Gerais da População Disponível em: http://www.ibge.gov.br/home/estatistica/ populacao/censo2000/populacao/ religiao_Censo2000.pdf

7. Rowe JW, Kahn RL. Successful aging. The Gerontologist 1997; 37 (4): 433-440.

8. Moraes JFD, Souza VBA. Factors associated with the successful aging of the socially-active elderly in the metropolitan region of Porto Alegre. Rev Bras Psiquiatr 2005; 27(4): 302-308.

9. Rosa LHT. Estudo dos fatores associados ao envelhecimento bem-sucedido de idosos da comunidade de Barra Funda-RS. Tese [ Doutorado apresentada ao Programa de PósGraduação] - Pontifícia Universidade Católica do Rio Grande do Sul ; 2008.

10. Wong PTP. Spirituality, meaning and successful aging. In: PTP Wong \& PS Fry (Eds.) The human quest for meaning: a handbook of psychological research and clinical applications. NJ: Lawrence Erlbaum Associates; 1998. 359-394.
11. Crowther MR, et al. Rowe and Kahn's Model of Successful Aging Revisited Positive Spirituality-The Forgotten Factor. The Gerontologist 2002; 42:613-620.

12. Vecchia RD, et al. Qualidade de vida na terceira idade: um conceito subjetivo. Rev bras epidemiol 2005; 8(3): 246-252.

13. Sawatzky R, Ratner PA, Chiu L. A MetaAnalysis of the Relationship Between Spirituality and Quality of Life. Social Indicators Research 2005; 72(2): 153-188.

14. Katsuno T. Personal Spirituality of Persons with Early-Stage Dementia. Dementia 2003, 2(3): 315335.

15. Spreitzer E, Snyder EE. Correlates of life satisfaction among the aged. Journal of Gerontology 1974; 29: 454-458.

16. Krause N. Measuring religiosity in later life. Research on aging 1993; 15: 170-197.

17. Wang $\mathrm{CW}$,et al. The impact of spirituality on health-related quality of life among Chinese older adults with vision impairment. Aging \& Mental Health 2008; 12(2): 267 - 275.

18. Doenças crônico-degenerativas e obesidade: estratégia mundial sobre alimentação saudável, atividade física e saude. [Acessado em $31 \mathrm{mar}$ 2009] Disponível em: http://www.opas.org.br/ publicmo.cfm? codigo $=66$.

19. McCauley J, et al. Daily spiritual experiences of older adults with and without arthritis and the relationship to health outcomes. Arthritis Care and Research 2007; 59(1): 122-128.

20. Bartlett SJ, et al. Spirituality, well-being, and quality of life in people with rheumatoid arthritis. Arthritis Rheum.2003 Dec 15;49(6):778-83.

21. Potter ML, Zauszniewski JA. Spirituality, resourcefulness, and arthritis impact on health perception of elders with rheumatoid arthritis. J Holist Nurs.2000;18(4):311-31.

22. Hummer RA, et al. Religious involvement and U.S adult mortality. Demography 1999; 36(2): 273-285.

23. Bekelman DB, et al. Spiritual Well-Being and Depression in Patients with Heart Failure. Journal of General Internal Medicine 2007; 22(4): 470-477.

24. Giaquinto S, Spiridigliozzi C, Caracciolo B. Can Faith Protect From Emotional Distress After Stroke? Stroke 2007;38:993-997 
25. Gillum RF, Ingram DD. Frequency of Attendance at Religious Services, Hypertension, and Blood Pressure: The Third National Health and Nutrition Examination Survey. Psychosomatic Medicine 2006, 68:382-385.

26. Stavig GR, Igra A, Leonard AR. Hypertension among Asians and pacific islanders in California. Am J Epidemiol 1984; 119(5): 677691.

27. Krause $\mathrm{N}$ et al. Religion, Death of a Loved One, and Hypertension Among Older Adults in Japan. The Journals of Gerontology Series B: Psychological Sciences and Social Sciences 2002; 57:S96S107.

28. Fehring RJ, Miller JF, Shaw C. Spiritual wellbeing, religiosity, hope, depression, and other mood states in elderly people coping with cancer. Oncology Nursing Forum 1997;24:663-671

29. Pressman P, et al. Religious belief, depression, and ambulation status in elderly women with broken hips. Am J Psychiatry 1990; 147:758-760.

30. Parker $\mathrm{M}$,et al. Religiosity and mental health in southern, community-dwelling older adults. Aging \& Mental Health 2003;7:390-397

31. Blay SL,et al. The Relationship Between Religiosity and Tobacco, Alcohol Use, and Depression in an Elderly Community Population. Am J Geriatric Psychiatry 2008; 16(11): 934-943.

32. Koenig HG. Religion and Depression in Older Medical Inpatients. Am J Geriatric Psychiatry 2007; 15(4): 282-291.

33. Beekman AT ,et al. Consequences of major and minor depression in later life: A study of disability, well-being and service utilization. Psychological Medicine 1997;27:1397-1409.

34. Koenig HG, George LK, Peterson BL. Religiosity and remission of depression in medically ill older patients. Am J Psycbiatry 1998;155:536-542

35. Tapanya S, Nicki R, Jarusawad O. Worry and intrinsic/extrinsic religious orientation among Buddhist (Thai) and Christian (Canadian) elderly persons. Int J Aging and Human Development 1997; 44: 73-83.

36. Baker M, Gorsuch R. Trait anxiety and intrinsic/extrinsic religiousness. Journal for the scientific Study of Religion 1982; 21: 119-122.

37. Kaufman $Y$,et al. Cognitive decline in Alzheimer disease. Impact of spirituality, religiosity, and QOL. Neurology 2007;68:15091514.
38. Hill TD ,et al. Religious Attendance and Cognitive Functioning Among Older Mexican Americans. J Gerontol Series B: Psychological Sciences and Social Sciences 2006, 61:P3-P9.

39. Smith LG. P2-262: Prayer as an intervention for agitated dementia residents. Alzheimer's and dementia 2008; 4(4-Sup 1): T448.

40. Khouzam HR, Smith CE, Bissett B. Bible therapy: a treatment of agitation in elderly patients with Alzheimer's disease. Clinical gerontologist 1994; 15(2): 71-74.

41. Vance DE. Spiritual Activities for Adults with Alzheimer's Disease The Cognitive Components of Dementia and Religion. Journal of Religion, Spirituality \& Aging 2005, 17(1/2): 109-130.

42. Bingham V, Habermann B. The influence of spirituality on family management of Parkinson's disease. 2006 Dec;38(6):422-7.

43. Pargament KI, et al. Religious Coping Methods as Predictors of Psychological, Physical and Spiritual Outcomes among Medically Ill Elderly Patients: A Two-year Longitudinal Study. J Health Psychology 2004; 9(6): 713-730.

44. Koenig HG, George LK, Titus P. Religion, Spirituality, and Health in Medically Ill Hospitalized Older Patients. J Am Geriatrics Society 2004; 52(4): 554-562.

45. Kim J, Heinemann AW. Spirituality, quality of life and functional recovery following medical rehabilitation: Paper presented at the $106^{\text {th }}$ annual convention of the American Psychological Association. São Francisco ;1998

46. Pargament KI, et al. Religious Struggle as a Predictor of Mortality Among Medically Ill Elderly Patients. Arch Internal Medicine 2001; 161(15): 1881-1885.

47. Chida Y, Steptoe A, Powell LH. Religiosity/ Spirituality and Mortality. A Systematic Quantitative Review. Psychother Psychosom 2009;78:81-90.

48. McCullough ME, Hoyt WT, Larson DB, Koenig HG. Religious involvement and mortality: a meta-analytic review. Health Psychology 2000; 19(3):211-222.

49. Hall DE. Religious Attendance: more CostEffective Than Lipitor? The Journal of the American Board of Family Medicine 2006; 19:103-109.

50. Murray SA ,et al. Exploring the spiritual needs of people dying of lung cancer or heart failure: a prospective qualitative interview study of patients and their carers. Palliative Medicine 2004;18 (1):39-45. 
51. Ehman JW,et al. Do Patients Want Physicians to Inquire About Their Spiritual or Religious Beliefs If They Become Gravely Ill? Archives of Internal Medicine 1999; 159(15): 1803-1806.

52. McClain CS, Rosenfeld B, Breitbart W. Effect of spiritual well-being on end-of-life despair in terminally-ill cancer patients. The Lancet 2003; 361(9369): 1603-1607.

53. Puchalski CM, Dorff RE, Hendi IY. Spirituality, religion, and healing in palliative care. Clinics in Geriatric Medicine 2004; 20(4): 689-714.

54. Lo B ,et al. Discussing religious and spiritual issues at the end of life: a practical guide for physicians. JAMA 2002; 13;287(6):749-54.

55. Peres MFP, et al. A importância da integração da espiritualidade e da religiosidade no manejo da dor e dos cuidados paliativos. Rev psiquiatr clín 2007, 34(supl. 1): 82-87.

56. Lucchetti G, Granero AL, Bassi RM, Latorraca $R$, Nacif SAP. Espiritualidade na prática clínica: o que o clínico deve saber? Rev Bras Clin Med 2010;8(2):154-8.

57. Sherman AC, et al. Religious Struggle and Religious Comfort in Response to Illness: Health Outcomes among Stem Cell Transplant Patients. Journal of Behavioral Medicine 2005; 28(4): 359-367.

58. Pargament KI, et al. Patterns of positive and negative religious coping with major life stressors : Symposium on religion, health, and well-being. Journal for the scientific study of religion 1998; 37(4): 710-724. 
